\title{
Full Graph Methods of Switched Current Circuit Solution
}

\author{
Bohumil Brtník \\ Department of the Electronics and Informatics, College of Polytechnics, Jihlava 58601, Czech Republic
}

Received: May 02, 2011 / Accepted: June 01, 2011 / Published: June 25, 2011.

\begin{abstract}
Circuits with switched current are described by an admittance matrix and seeking current transfers then means calculating the ratio of algebraic supplements of this matrix. As there are also graph methods of circuit analysis in addition to algebraic methods, it is clearly possible in theory to carry out an analysis of the whole switched circuit in two-phase switching exclusively by the graph method as well. For this purpose it is possible to plot a Mason graph of a circuit, use transformation graphs to reduce Mason graphs for all the four phases of switching, and then plot a summary graph from the transformed graphs obtained this way. First the author draws nodes and possible branches, obtained by transformation graphs for transfers of EE (even-even) and OO (odd-odd) phases. In the next step, branches obtained by transformation graphs for EO and OE phase are drawn between these nodes, while their resulting transfer is multiplied by $z^{-\frac{1}{2}}$. This summary graph is extended by two branches from input node and to output node, the extended graph can then be interpreted by the Mason's relation to provide transparent current transfers. Therefore it is not necessary to compose a sum admittance matrix and to express this consequently in numbers, and so it is possible to reach the final result in a graphical way.
\end{abstract}

Key words: Switched current circuits, two phases, transformation graph, Mason's formula, current transfer, summary MC-graph.

\section{Introduction}

Circuits with switched currents are described by an admittance matrix and seeking current transfers then means calculating the ratio of algebraic supplements of this matrix [1]. As there are also graph methods of circuit analysis in addition to algebraic methods [2], it is clearly possible in theory to carry out an analysis of the whole switched circuit in two-phase switching exclusively by the graph method as well.

In this paper, this method will be demonstrated in the example. The summa MC-graph is constructed using transformation graphs in two-phase switching. Graph methods give results in a symbolic form by the Mason's relation [3], which makes it possible to use them to find general relations, too.

Switching phases are marked as odd and even not 1 and 2, the nodes are numbered.

The paper is organized as follows: Section 2 describes construction of the transformation graph of the switch and its evaluation; section 3 presents

Corresponding author: Bohumil Brtník, Ph.D., research field: theory of electronics circuits. E-mail: brtnik@vspj.cz. construction of the summary MC-graph based on transformation graphs, which contained all four phases; section 4 gives general rules for summary MC-graph construction; section 5 describes calculation of the transmissions of the currents from the extended summary MC-graph; section 6 gives example of a full-graph solution of the circuit by the described method of a summary MC-graph constructed on the transformation graphs; results are in a symbolic form; section 7 gives conclusions.

\section{Construction of the Transformation Graph}

The circuit with a switch whose circuit diagram is in Fig. 1 can be described by the nodal method [4] to an equation system (1).

$$
\begin{aligned}
& \tilde{I}_{1}=y_{1} \cdot \tilde{V}_{1}+y_{3} \cdot\left(\tilde{V}_{1}-\tilde{V}_{2}\right) \\
& \tilde{I}_{2}=y_{2} \cdot \tilde{V}_{2}+y_{3} \cdot\left(\tilde{V}_{2}-\tilde{V}_{1}\right)
\end{aligned}
$$

After switching nodes 1 and 2 are connected, as shown in Fig. 2, and the circuit diagram can be described by the nodal voltage method [5] by Eq. (2).

$$
I=\left(y_{1}+y_{2}\right) \cdot V
$$




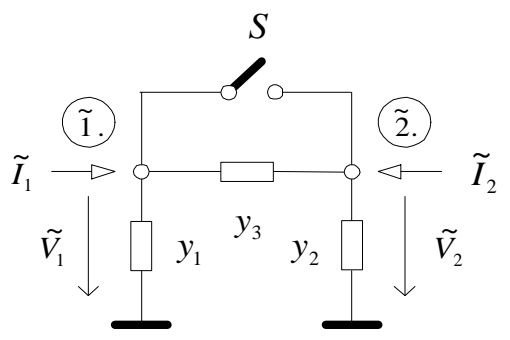

Fig. 1 Schematic diagram simplest of the circuit with a switch.

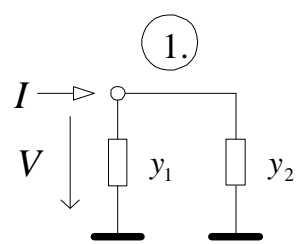

Fig. 2 Schematic diagram after switching.

Closing the switch is manifested by connecting nodes 1 and 2, and thus by uniting the voltages $\tilde{V}_{1}$ and $\tilde{V}_{2}$, which is described by the set of Eqs. (3) after rewriting (4), where $V$ is the resulting voltage.

$$
\begin{aligned}
& \tilde{V}_{1}=V \\
& \tilde{V}_{2}=V
\end{aligned}
$$

Secondly current $I$ is described by the following Eq. (4):

$$
I=\tilde{I}_{1}+\tilde{I}_{2}
$$

The system of Eqs. (3) and (4) can be rewritten in the following transformation of Eqs. (5):

$$
\begin{aligned}
& \tilde{V}_{1}=1 . V \\
& \tilde{V}_{2}=1 . V \\
& I=1 . \tilde{I}_{1}+1 . \tilde{I}_{2}
\end{aligned}
$$

This set of transformation Eqs. (5) can be represented graphically as a voltage transformation graph and a current transformation graph [6], thus the transformation graph (T-graph), as it is shown in Fig. 3. The branches of the transformation graph with the transfers of voltage are marked as $\rightarrow$ and those of current as $\rightarrow$.

The algorithm of the evaluation of the transformation graph will be explained by comparing MC-graphs before and after transformation as it is shown in Fig. 4, where the MC-graph of the set of equations before transformation (1) is at the top and the
MC-graph of the Eq. (2) after transformation (transformed graph) is at the bottom.

Since the set of Eqs. (5) can be rewritten in the following general form $\tilde{I}=\tilde{y} \cdot \tilde{V}, I=y \cdot V, I=a_{I} \cdot \tilde{I}$ and $\tilde{V}=a_{V} \cdot V$, then it will hold $I=a_{I} \cdot \tilde{I}=a_{I} \cdot \tilde{y} \cdot \tilde{V}=a_{I} \cdot \tilde{y} \cdot a_{V} \cdot V=y \cdot V$. The admittance in the resulting node is given by the relation $a_{I} \cdot \tilde{y} \cdot a_{V}=y$, generally

$$
a_{I} \cdot \tilde{y} \cdot a_{V} \cdot \alpha=y
$$

Eq. (6) can be represented graphically, as it is shown in Fig. 5.

As we can see, to eliminate the variable $y_{3}$ from the resulting relationship $y_{1}+y_{2}$, the coefficient $\alpha$ must be negative. Thus the general relation $a_{I} \cdot \tilde{y} \cdot a_{V} \cdot \alpha=y$ will be

$$
\begin{array}{r}
\left(1 \cdot y_{1} \cdot 1 \cdot 1+1 \cdot y_{3} \cdot 1 \cdot 1\right)+\left(1 \cdot y_{2} \cdot 1 \cdot 1+1 \cdot y_{3} 1 \cdot 1\right) \\
+1 \cdot y_{3} \cdot 1 \cdot(-1)+1 \cdot y_{3} \cdot 1 \cdot(-1)=y_{1}+y_{2}
\end{array}
$$

where $a_{I}=1, a_{V}=1$. As the branch of the original MC-graph converts to the inherent loop in the resulting transformed graph, the coefficient $\alpha=-1$.

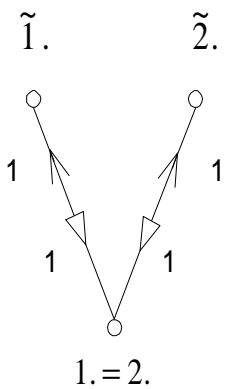

Fig. 3 Transformation graph of the switch.

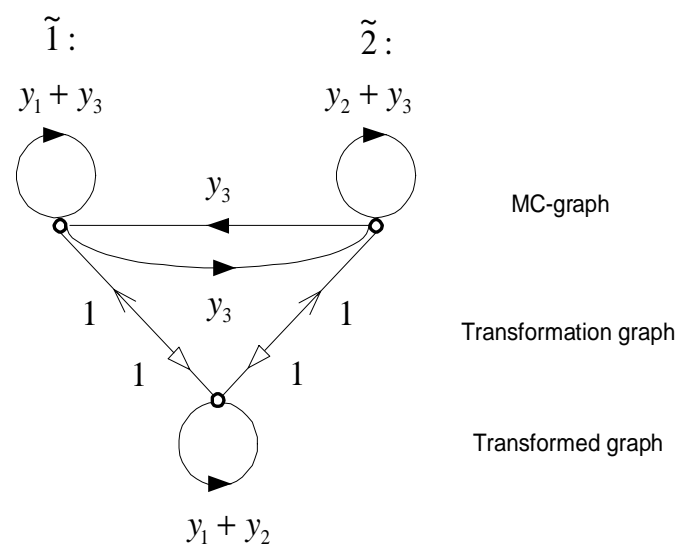

Fig. 4 Evaluation of the transformation graph. 


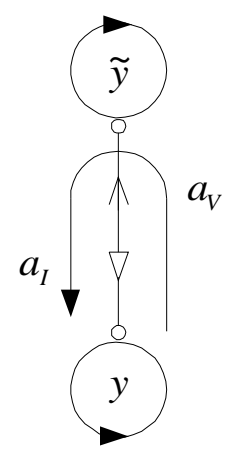

Fig. 5 Graph of the Eq. (6).

\section{Summary MC-graph Construction Based on Transformation Graph}

The circuit with switched currents whose circuit diagram is in Fig. 6 can be described by the nodal method by the equation system (8).

$$
\begin{aligned}
& I_{1 E}(t)=+y^{(1 .)} \cdot\left[V_{1 E}(t)-V_{2 E}(t)\right]+y^{(2 .)} \cdot\left(V_{1 O}-V_{2 O}\right) \\
& I_{2 E}(t)=-y^{(1 .)} \cdot\left[V_{1 E}(t)-V_{2 E}(t)\right]-y^{(2 .)} \cdot\left(V_{1 O}-V_{2 O}\right) \\
& I_{1 O}(t)=+y^{(1 .)} \cdot\left(V_{1 E}-V_{2 E}\right)+y^{(2 .)} \cdot\left[V_{1 O}(t)-V_{2 O}(t)\right] \\
& I_{2 O}(t)=-y^{(1 .)} \cdot\left(V_{1 E}-V_{2 E}\right)-y^{(2 .)} \cdot\left[V_{1 O}(t)-V_{2 O}(t)\right]
\end{aligned}
$$

This system of Eqs. (8) can be rewritten in the following form (9):

$$
\begin{aligned}
& I_{1 E}(t)=+y^{(1 .)} \cdot V_{1 E}(t)-y^{(1 .)} \cdot V_{2 E}(t)+y^{(2 .)} \cdot V_{1 O}-y^{(2 .)} \cdot V_{2 O} \\
& I_{2 E}(t)=-y^{(1 .)} \cdot V_{1 E}(t)+y^{(1 .)} \cdot V_{2 E}(t)-y^{(2 .)} \cdot V_{1 O}+y^{(2 .)} \cdot V_{2 O}(9) \\
& I_{1 O}(t)=+y^{(1 .)} \cdot V_{1 E}-y^{(1 .)} \cdot V_{2 E}+y^{(2 .)} \cdot V_{1 O}(t)-y^{(2 .)} V_{2 O}(t) \\
& I_{2 O}(t)=-y^{(1 .)} \cdot V_{1 E}+y^{(1 .)} \cdot V_{2 E}-y^{(2 .)} \cdot V_{1 O}(t)+y^{(2 .)} \cdot V_{2 O}(t)
\end{aligned}
$$

If the difference between the even phase and the odd phase is expressed by means of the Z-transformation by multiplying by the $z^{-\frac{1}{2}}$ operator, it is then possible to rewrite the equation system (9) to the form (10).

$$
\begin{aligned}
& I_{1 E}=+y^{(1 .)} \cdot V_{1 E}-y^{(1 .)} \cdot V_{2 E}+z^{-\frac{1}{2}} y^{(2 .)} \cdot V_{1 O}-z^{-\frac{1}{2}} y^{(2 .)} \cdot V_{2 O} \\
& I_{2 E}=-y^{(1 .)} \cdot V_{1 E}+y^{(1 .)} \cdot V_{2 E}-z^{-\frac{1}{2}} y^{(2 .)} \cdot V_{1 O}+z^{-\frac{1}{2}} y^{(2 .)} \cdot V_{2 O} \\
& I_{1 O}=+z^{-\frac{1}{2}} y^{(1 .)} \cdot V_{1 E}-z^{-\frac{1}{2}} y^{(1 .)} \cdot V_{2 E}+y^{(2 .)} \cdot V_{1 O}-y^{(2 .)} V_{2 O} \\
& I_{2 O}=-z^{-\frac{1}{2}} y^{(1 .)} \cdot V_{1 E}+z^{-\frac{1}{2}} y^{(1 .)} \cdot V_{2 E}-y^{(2 .)} \cdot V_{1 O}+y^{(2 .)} \cdot V_{2 O}
\end{aligned}
$$

The equation system (10) can be written e.g. in the following generally form (11), where admittance are generally marked $y_{i j}$.

$$
\begin{aligned}
& 0=+y_{11} \cdot V_{1 E}-y_{12} \cdot V_{2 E}+z^{-\frac{1}{2}} y_{13} \cdot V_{1 O}-z^{-\frac{1}{2}} y_{14} \cdot V_{2 O} \\
& 0=-y_{21} \cdot V_{1 E}+y_{22} \cdot V_{2 E}-z^{-\frac{1}{2}} y_{23} \cdot V_{1 O}+z^{-\frac{1}{2}} y_{24} \cdot V_{2 O} \\
& 0=+z^{-\frac{1}{2}} y_{31} \cdot V_{1 E}-z^{-\frac{1}{2}} y_{32} \cdot V_{2 E}+y_{33} \cdot V_{1 O}-y_{34} V_{2 O} \\
& 0=-z^{-\frac{1}{2}} y_{41} \cdot V_{1 E}+z^{-\frac{1}{2}} y_{42} \cdot V_{2 E}-y_{43} \cdot V_{1 O}+y_{44} \cdot V_{2 O}
\end{aligned}
$$

Fig. 6 Schematic diagram of a switched current circuit.

If the first constituent is expressed from first equation and the second constituent from the second, etc., the Eqs. (11) will get the form (12).

$$
\begin{aligned}
& y_{11} \cdot V_{1 E}=y_{12} \cdot V_{2 E}-z^{-\frac{1}{2}} y_{13} \cdot V_{1 O}+z^{-\frac{1}{2}} y_{14} \cdot V_{2 O} \\
& y_{22} \cdot V_{2 E}=y_{21} \cdot V_{1 E}+z^{-\frac{1}{2}} y_{23} \cdot V_{1 O}-z^{-\frac{1}{2}} y_{24} \cdot V_{2 O} \\
& y_{33} \cdot V_{1 O}=-z^{-\frac{1}{2}} y_{31} \cdot V_{1 E}+z^{-\frac{1}{2}} y_{32} \cdot V_{2 E}+y_{34} \cdot V_{2 O} \\
& y_{44} \cdot V_{2 O}=+z^{-\frac{1}{2}} y_{41} \cdot V_{1 E}-z^{-\frac{1}{2}} y_{42} \cdot V_{2 E}+y_{43} \cdot V_{1 O}
\end{aligned}
$$

Two parts of this Eqs. (12), which are (13)

$$
\begin{array}{ll}
y_{11} \cdot V_{1 E}=y_{12} \cdot V_{2 E}, & y_{33} \cdot V_{1 O}=y_{34} \cdot V_{2 O} \\
y_{22} \cdot V_{2 E}=y_{21} \cdot V_{1 E} & y_{44} \cdot V_{2 O}=y_{43} \cdot V_{1 O}
\end{array}
$$

can be demonstrated by MC-graphs. The construction of corresponding MC-graphs is following: The first constituent is expressed from each first equation and the second constituent from the second ones. Graph interpretation of Eqs. (13) for e.g. the last equation is that the constituent $y_{44} \cdot V_{20}$, represented by the voltage of the $2 \mathrm{O}$ node multiplied by the coefficient $y_{44}$ (which means that the $2 \mathrm{O}$ node's own loop has the transfer $\left.y_{44}\right)$, is given by the constituent $y_{43} \cdot V_{10}$, i.e., by the addition of $y_{43}$ from the 10 node (therefore the branch going from the $1 \mathrm{O}$ node to the $2 \mathrm{O}$ node has the transfer $\left.y_{43}\right)$. 
Described construction is shown in Fig. 7.
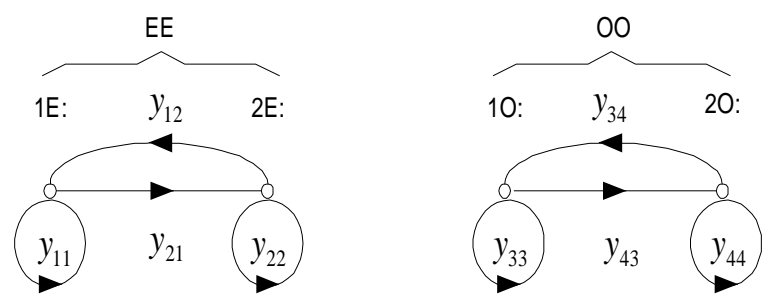

Fig. 7 MC-graph for the same phase.

The principle of superposition implies that the total effect is given by the sum of effects; hence we have to add to the graph the remaining effect, described by the set of Eqs. (14).

$$
\begin{aligned}
& y_{11} \cdot V_{1 E}=-z^{-\frac{1}{2}} y_{13} \cdot V_{1 O}+z^{-\frac{1}{2}} y_{14} \cdot V_{2 O} \\
& y_{22} \cdot V_{2 E}=+z^{-\frac{1}{2}} y_{23} \cdot V_{1 O}-z^{-\frac{1}{2}} y_{24} \cdot V_{2 O} \\
& y_{33} \cdot V_{1 O}=-z^{-\frac{1}{2}} y_{31} \cdot V_{1 E}+z^{-\frac{1}{2}} y_{32} \cdot V_{2 E} \\
& y_{44} \cdot V_{2 O}=+z^{-\frac{1}{2}} y_{41} \cdot V_{1 E}-z^{-\frac{1}{2}} y_{42} \cdot V_{2 E}
\end{aligned}
$$

Next, from e.g. the last equation in the system (14), the form (15) will thus be obtained.

$$
\begin{gathered}
y_{44} \cdot V_{2 O}=z^{-\frac{1}{2}} y_{41} \cdot V_{1 E}-z^{-\frac{1}{2}} y_{42} \cdot V_{2 E}+y_{43} \cdot V_{1 O} \\
=y_{43} \cdot V_{1 O}+\left[z^{-\frac{1}{2}} y_{41} \cdot V_{1 E}-z^{-\frac{1}{2}} y_{42} \cdot V_{2 E}\right]
\end{gathered}
$$

However the first constituent $y_{43} \cdot V_{1 O}$ of the Eq. (15) has already been demonstrated by the graph in Fig. 7 as it is the last equation from the system (13). The principle of superposition implies that the total effect is given by the sum of effects; hence we have to add to the graph the remaining effect given in this case by the relation $z^{-\frac{1}{2}} y_{41} \cdot V_{1 E}-z^{-\frac{1}{2}} y_{42} \cdot V_{2 E}$ which indicates in general the addition of the remaining elements. The graph representation of the second constituent $z^{-\frac{1}{2}} y_{41} \cdot V_{1 E}-z^{-\frac{1}{2}} y_{42} . V_{2 E}$, i.e., Eq. (16) is then following:

$$
y_{44} \cdot V_{2 O}=+z^{-\frac{1}{2}} y_{41} \cdot V_{1 E}-z^{-\frac{1}{2}} y_{42} \cdot V_{2 E}
$$

The left side of the equation, i.e., the constituent $y_{44} \cdot V_{2 O}$, represents the voltage of node $2 O$ (i.e., the voltage of the second node in the odd phase of $V_{20}$ ), with the own loop of the node $2 O$ with the transfer $y_{22}$. This constituent $y_{44} \cdot V_{2 O}$ is given by the sum of constituents $z^{-\frac{1}{2}} y_{41} \cdot V_{1 E}$, representing the addition of $z^{-\frac{1}{2}} \cdot y_{41}$ from the node $V_{1 E}$, which means that the branch going from the node $1 \mathrm{E}$ to the node $2 \mathrm{O}$ has the transition $z^{-\frac{1}{2}} y_{41}$. Further it is given by the constituent $-z^{-\frac{1}{2}} y_{42} . V_{2 E}$, representing the addition of $-z^{-\frac{1}{2}} y_{42}$ from the node $V_{2 E}$, which means that the branch going from the node $2 \mathrm{E}$ to the node $2 \mathrm{O}$ has the transition $-z^{-\frac{1}{2}} y_{42}$.

The above described facts are illustrated by the graph in Fig. 8.

\section{Rules for Summary Graph Construction}

Rules for summary graph construction can now be easily formulated by generalizing the construction described in the previous paragraph this way:

First we draw the nodes' own loops (and branches when applicable) as the results from the transformation graph for the even phase (EE) and the own loops (and branches when applicable) as the results from the transformation graph for the odd phase (OO);

Between thus obtained nodes and branches, we will consequently draw branches as the results from the transformation graph for the opposite phase, whose transfer obtained by evaluating the transformation graph is multiplied by the coefficient $-z^{-\frac{1}{2}}$ if the branch connects nodes of the same name and by the coefficient $+z^{-\frac{1}{2}}$ in other cases.

Thus the graph representation of the Eqs. (14) is in Fig. 9.

\section{Calculation of the Transmissions from the Summary Graph}

Description by the nodal method leads to an equation system, which can have the following form, for instance (17): 


$$
\begin{aligned}
& I_{1}=y_{11} \cdot V_{1}+y_{12} \cdot V_{2} \\
& I_{2}=y_{21} \cdot V_{1}+y_{22} \cdot V_{2}
\end{aligned}
$$

This system can be rewritten as (18)

$$
\begin{aligned}
& y_{11} \cdot V_{1}=1 . I_{1}-y_{12} \cdot V_{2} \\
& y_{22} \cdot V_{2}=1 . I_{2}-y_{22} \cdot V_{2}
\end{aligned}
$$

and illustrated by an MC-graph, which is shown in Fig. 10 .

For the calculation of the current transfer it is necessary to modify the graph.

Output current is described by the equation $I_{2}=y_{22} . V_{2}$, which can be rewritten as $1 . I_{2}=y_{22} \cdot V_{2}$ and illustrated by an MC-graph, which is shown in Fig. 11.

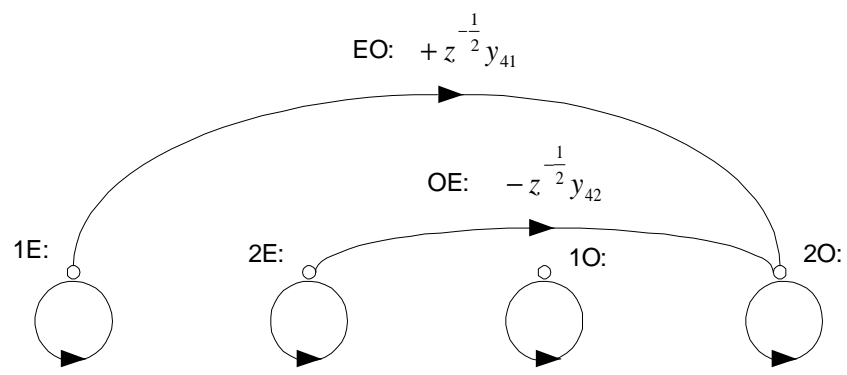

Fig. 8 MC-graph of the Eq. (16).

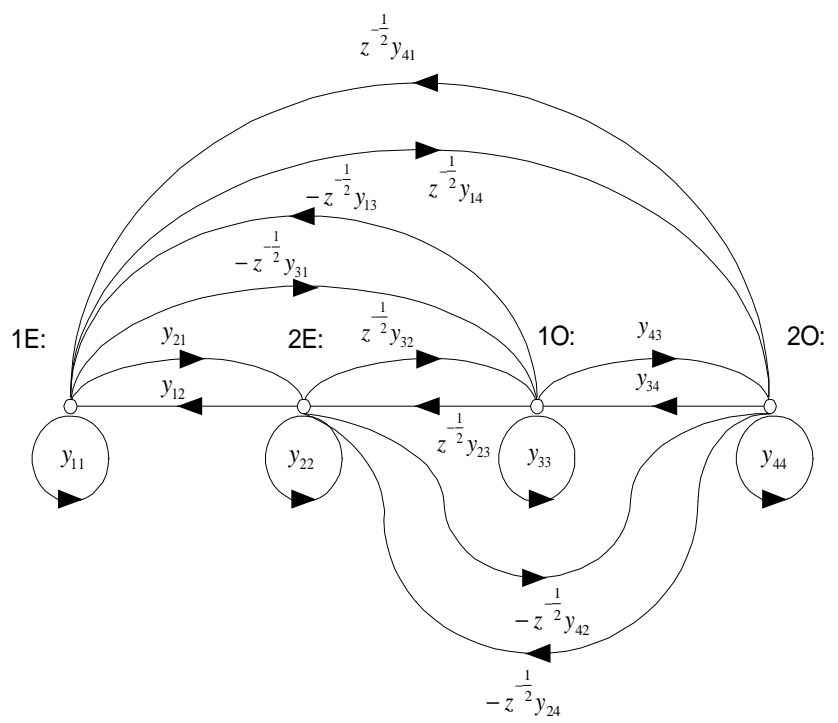

Fig. 9 MC-graph set of Eqs. (14).

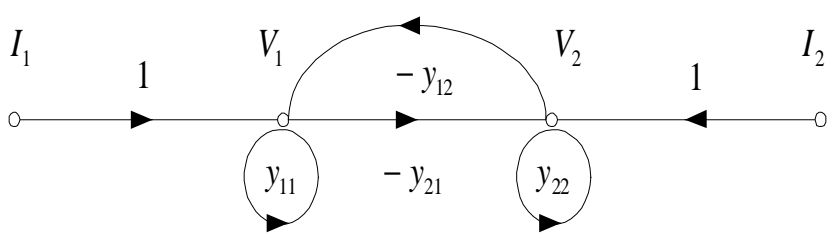

Fig. 10 Graph of the set of Eqs. (18).

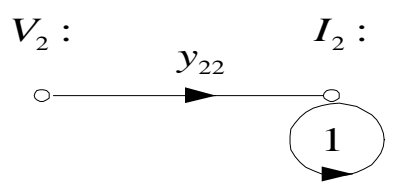

Fig. 11 MC-graph of the equation $1 . \mathrm{I}_{2}=\mathrm{y}_{22} \cdot \mathrm{V}_{2}$.

The current transfers will now be obtained from an extended graph, i.e., the graph must be extended to two branches as it is shown in Fig. 12. The first branch from the input node $\mathrm{I}_{\mathrm{INP}}$ to the node $1 \mathrm{E}$ with transfer 1 and the second branch from the node $2 \mathrm{E}$ to the node $\mathrm{I}_{\mathrm{OUT}}$, the transfer is equal to the transmission of its own loop at the output node.

The summary graph is then evaluated by means of the Mason's rule $T=\frac{I_{\text {OUT }}}{I_{I N P}}=\frac{\sum p_{(i)} \cdot \Delta_{(i)}}{V-\sum S^{(K)} \cdot V^{(K)}}$.

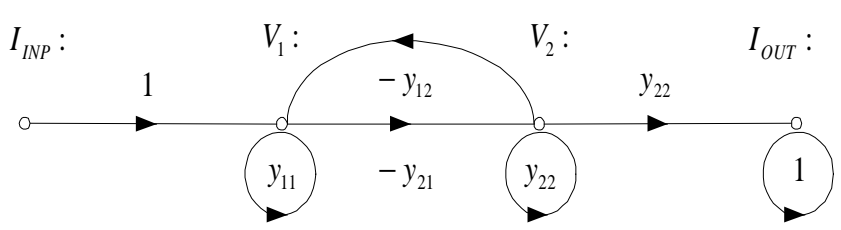

Fig. 12 Extended MC-graph of the SI circuit from the solution.

\section{Example of Full-Graph Solution}

A solution of a circuit by the described method of a summary MC-graph constructed on the transformation graph will be shown by solving a circuit with three field effect transistors and two capacitors [3], whose wiring diagram is in Fig. 13.

The field effect transistor is described by the equation $I_{D}=y_{21} \cdot V_{G}+y_{22} \cdot V_{D}$, this equation can be

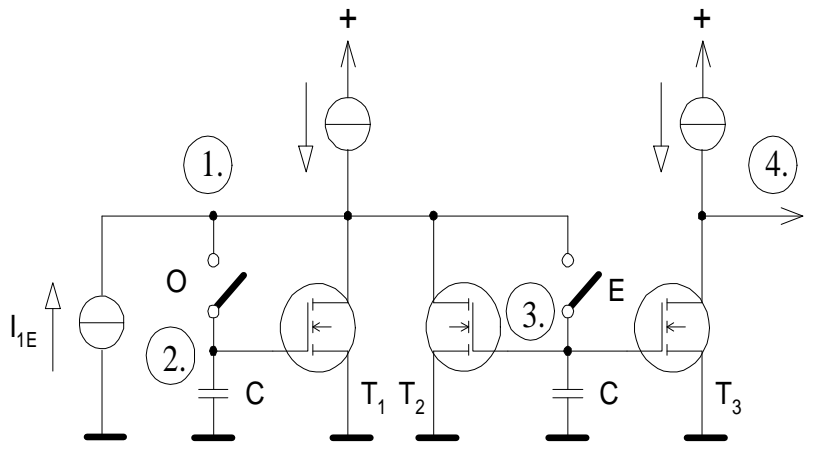

Fig. 13 Schematic diagram of the SI circuit for the example. 
rewritten in the following form $y_{22} \cdot V_{D}=-y_{21} \cdot V_{G}+I_{D}$, if $I_{D}=0$ then $y_{22} \cdot V_{D}=-y_{21} \cdot V_{G}$.

The graph representation of this equation is in Fig. 14. The left side of the equation $y_{22} \cdot V_{D S}$ represents the own loop of the node $D$ with the transfer $y_{22}$, the right side branch going from the node $G$ to the node $D$ has the transition $y_{21}$.

The circuit in Fig. 13 has four nodes; therefore the starting graph of the circuit in Fig. 15 has also four nodes.

Drain $\mathrm{D}$ of the transistor $\mathrm{T}_{1}$ is connected to the node 1 , gate $\mathrm{G}$ to the node 2 . Therefore, the transistor $T_{1}$ is represented by the branch $-y_{21}^{(1 .)}$ from node 2 to node 1 and its inherent loop $y_{22}^{(1 .)}$ at the first node. Drain D

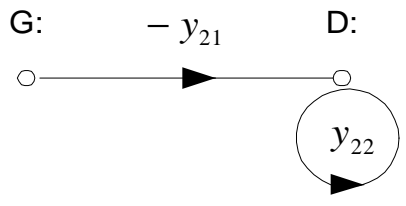

Fig. 14 MC-graph of the FET.
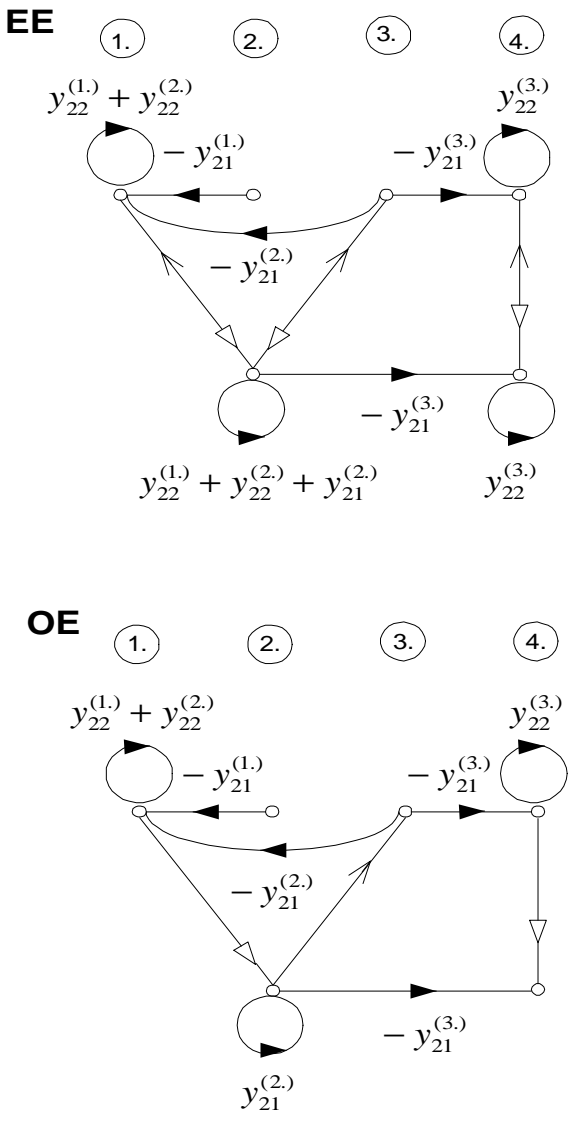

Fig. 15 Transformation graphs for EE, OO, EO and OE phases. of the transistor $T_{2}$ is connected to the node 1 , gate $G$ to the node 3 . Therefore, the transistor $T_{2}$ is represented by the branch $-y_{21}^{(2 .)}$ from node 3 to node 1 and its inherent loop $y_{22}^{(2 .)}$ at the first node. Drain D of the transistor $T_{3}$ is connected to the node 4 , gate $G$ to the node 3 . Therefore, the transistor $\mathrm{T}_{3}$ is represented by the branch $-y_{21}^{(3 .)}$ from node 3 to node 4 and its inherent loop $y_{22}^{(3 .)}$ at the fourth node.

The branch between the nodes 3 and 1 with the transfer $-y_{21}^{(2 .)}$ is transformed to the inherent loop with the transfer $y_{21}^{(2 .)}$, because in the relation $a_{I} \cdot \tilde{y} \cdot a_{V} \cdot \alpha=y$ now $\alpha=-1$, as the branch of the original graph converts to the inherent loop in the resulting transformed graph. The inherent loop with the transfer $y_{22}^{(1 .)}+y_{22}^{(2 .)}$ is transformed to the inherent loop, too. Because now $\alpha=1$, the transfer is $y_{22}^{(1 .)}+y_{22}^{(2 .)}$, too. Therefore, the resulting transfer of its inherent loop is $y_{22}^{(1 .)}+y_{22}^{(2 .)}+y_{21}^{(2 .)}$.
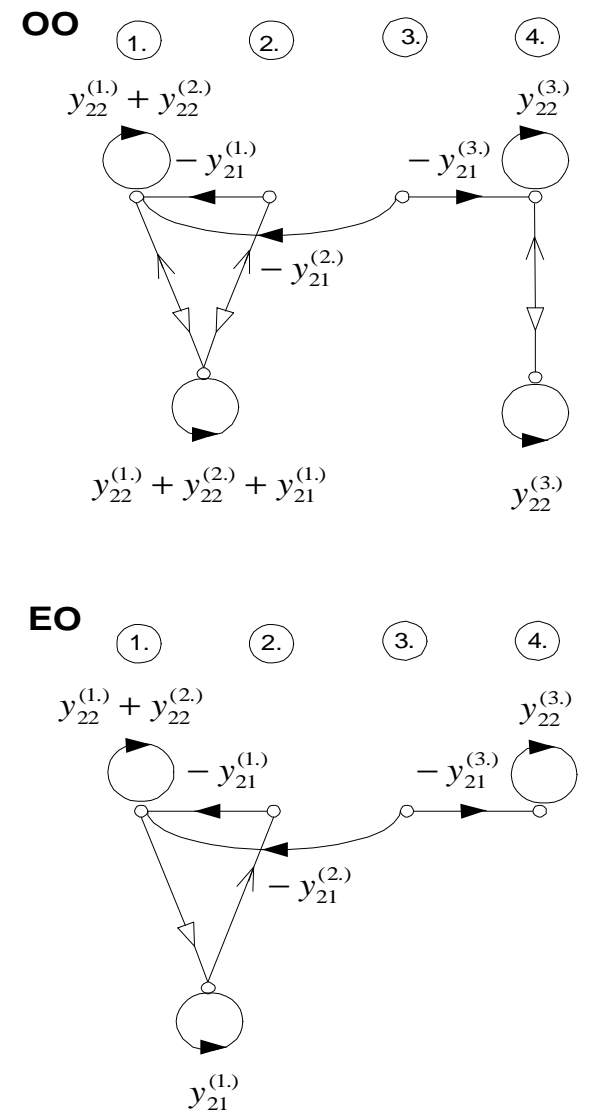


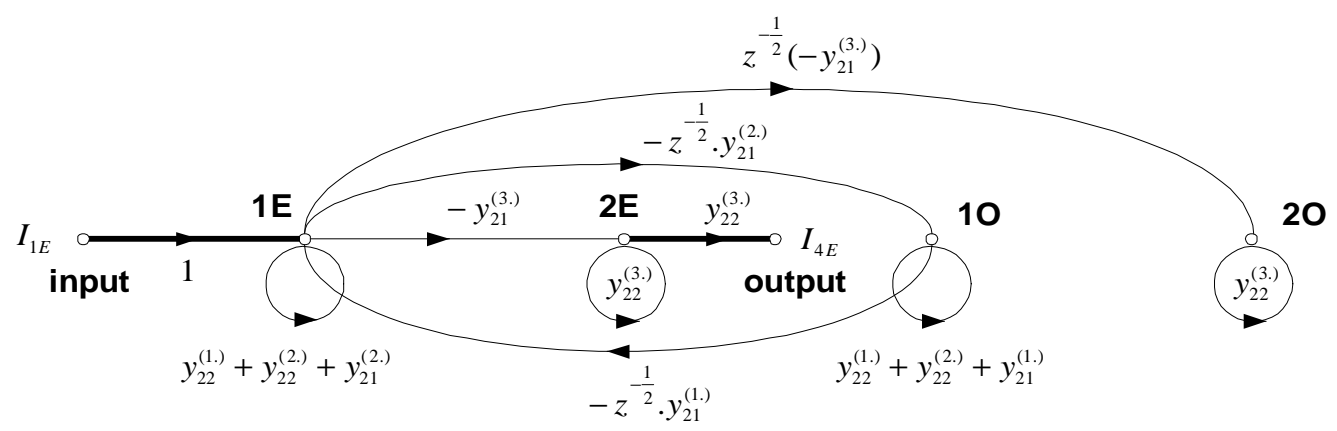

Fig. 16 The summary extended MC-graph.

The summary MC-graph obtained from the partial transformed graphs from Fig. 15 by the above described procedure is then shown in Fig. 16. First the results of the transformed graphs for $\mathrm{EE}$ and $\mathrm{OO}$ phases are plotted as nodes with the inherent loops with transfers $y_{22}^{(1 .)}+y_{22}^{(2 .)}+y_{21}^{(2 .)}, \quad y_{22}^{(3 .)}, \quad y_{22}^{(1 .)}+y_{22}^{(2 .)}+y_{21}^{(1 .)}$ and $y_{22}^{(3 .)}$ and one branch with transfer $-y_{21}^{(3 .)}$.

In the next step, the results of the transformed graph for the OE and EO phases multiplied by $\pm z^{-\frac{1}{2}}$ are then drawn between these nodes as branches, i.e., the branch with the transfer $z^{-\frac{1}{2}}\left(-y_{21}^{(3 .)}\right)$ between the nodes $1 \mathrm{E}$ and $2 \mathrm{O}$, the branch with the transfer $-z^{-\frac{1}{2}} y_{21}^{(2 .)}$ between the nodes $1 \mathrm{E}$ and $1 \mathrm{O}$, and the branch with the transfer $-z^{-\frac{1}{2}} y_{21}^{(1 .)}$ between the nodes $1 \mathrm{O}$ and 2E.

The current transfer $\frac{I_{4 E}}{I_{1 E}}$ will now be obtained from an extended graph, i.e., a graph must be extended to two branches: the first branch from the input node $\mathrm{I}_{\mathrm{INP}}$ $\left(I_{I N P}=I_{1 E}\right)$ to the node $1 \mathrm{E}$ with transfer 1 and the second branch from the node $2 \mathrm{E}$ to the node $\mathrm{I}_{\text {OUT }}$. $\left(I_{4 E}=I_{\text {OUT }}\right)$. The transfer is equal to the transmission of its own loop at the output node $2 \mathrm{E}$, i.e., $y_{22}^{(3 .)}$. The summary MC-graph is now evaluated by means of the Mason's rule, the current transfer $\frac{I_{4 E}}{I_{1 E}}$ is Eq. (19).

$$
\begin{gathered}
\frac{I_{\text {OUT }}}{I_{I N P}}=\frac{I_{4 E}}{I_{1 E}}=\frac{\sum p_{(i)} \cdot \Delta_{(i)}}{V-\sum S^{(K)} \cdot V^{(K)}}= \\
\frac{1\left(-y_{21}^{(3 .)}\right) y_{22}^{(3 .)}\left(y_{22}^{(1 .)}+y_{22}^{(2 .)}+y_{21}^{(1 .)}\right) y_{22}^{(3 .)}}{\left(y_{22}^{(1 .)}+y_{22}^{(2 .)}+y_{21}^{(2 .)}\right) y_{22}^{(3 .)}\left(y_{22}^{(1 .)}+y_{22}^{(2 .)}+y_{21}^{(1 .)}\right) y_{22}^{(3 .)}-z^{-\frac{1}{2}} y_{21}^{(2 .)} z^{-\frac{1}{2}} y_{21}^{(1 .)} y_{22}^{(3 .)} y_{22}^{(3 .)}}= \\
\frac{-y_{21}^{(3 .)}\left(y_{22}^{(1 .)}+y_{22}^{(2)}+y_{21}^{(1 .)}\right)}{\left(y_{22}^{(1 .)}+y_{22}^{(2)}+y_{21}^{(2)}\right)\left(y_{22}^{(1 .)}+y_{22}^{(2)}+y_{21}^{(1)}\right)-z^{-1} y_{21}^{(2)} y_{21}^{(1 .)}}
\end{gathered}
$$

\section{Conclusions}

There is described the theory of full graph solution of switched currents circuits in two phase switching. The theory is illustrated by an example solved, which is commented on in detail.

As shown, the solution is possible without the matrix calculus, only by graph method. Thanks to its clarity, the graphic method is extremely suitable even for understanding of these networks. A clearly arranged set of transformation graphs derived for different types of switching circuits can be used for analyzing switched networks and of course for understanding them, too. 
The M-C signal flow graphs can be used to analyze periodically switched linear circuits, too.

Graph methods give results in a symbolic form, which makes them possible to use to find general relations.

This full graph method can be used for solving SC circuits after its modification.

\section{References}

[1] D. Biolek, Solving Electronics Circuits, BEN Publisher, Prague, 2004.

[2] T. Dostál, The analysis of the active components containing switched capacitors by nodal voltage method, Electronics Horizont 45 (1984) 21-26.

[3] P. Martinek, P. Boreš, J. Hospodka, Electrics Filters, CVUT Publisher, Prague, 2003.

[4] C. Toumazou, Circuits and Systems Tutorials, IEEE Press Inc., New York, 1996.

[5] J. Vlach, K. Singhal, Computer Methods for Circuit Analysis and Design, Van Nostrand Reynhold, New York, 1994.

[6] D. Biolek, V. Biolkova, Analysis of circuits containing active elements by using modified t-graphs, in: N.E. Mastorakis, L.A. Pecorelli-Peres (Eds.), Advances in Systems Science: Measurement, Circuits and Control, WSEAS Press, Electrical and Computer Engineering Series, 2001, pp. 279-283. 